\title{
Die deutsche Wasserwirtschaft im europäischen Vergleich
}

\author{
Wasserwirtschaft; Regulierung; Liberalisierung; Privatisierung
}

Im Gegensatz zu anderen Infrastrukturbereichen ist die Wasserversorgung in Deutschland noch ein wettbewerbsrechtlicher Ausnahmebereich. Trotz einzelner Privatisierungen kommunaler Wasserversorgungsunternehmen findet kein Wettbewerb im Sinne einer Liberalisierung des Marktes statt. Deren konkrete Ausgestaltung hängt davon ab, welchen Regulierungen der Markt für Trinkwasser unterliegt. Im internationalen Vergleich sind drei Grundmodelle für die Regulierung von natürlichen Monopolen in der öffentlichen Wasserversorgung zu unterscheiden: das angelsächsische, das französische und das deutsche Modell. Dabei ist das Ausmaß der Abgrenzung zwischen Aufsichtsorganen und operativem Geschäft in England und Wales am höchsten und in Deutschland am niedrigsten. Daraus leiten sich drei Grundtypen der Privatisierung ab: „,Vollprivatisierung“, Privatisierung durch Delegation“ und „,Privatisierung mit Regulierung durch die Aufsichtsorgane", welche wiederum zu drei klar unterscheidbaren Formen des Wettbewerbs führen: Durch die Regulierungsbehörden simulierter Ersatzwettbewerb (,, Yardstick Competition “), Wettbewerb um zeitlich befristete Konzessionen für den Betrieb der Wasserversorgungsanlagen (,,Franchise Bidding“) und Wettbewerb auf den mit der Wasserversorgung verbundenen Märkten für Anlagen, Maschinen und Dienstleistungen der Wasserindustrie.

\section{I. Ökonomische Charakteristika der Wasserversorgung}

Während sich der Begriff der Privatisierung auf die Eigentumsverhältnisse der Versorgungsunternehmen bezieht, impliziert der Begriff der Liberalisierung übergeordnete ordnungspolitische Vorstellungen. Privatisierung beinhaltet die Ausgliederung öffentlicher Dienstleistungen aus dem öffentlichen Haushalt und Übertragung auf einen privatwirtschaftlich organisierten Träger. ${ }^{1}$ Dadurch muss sich aber an der Marktform und an der Wettbewerbsintensität für das entsprechende Gut nicht notwendigerweise etwas ändern. Im Rahmen der Privatisierung kann auch lediglich ein öffentliches Monopol in ein privates Monopol übergeführt werden. Die Liberalisierung bezieht sich darüber hinaus auf die ordnungspolitischen Rahmenbedingungen, sie bedeutet Wegfall von Wettbewerbsbeschränkungen und Versorgungsmonopolen und offenen Wettbewerb zwischen mehreren Anbietern um die Verbraucher.

Bei der Trinkwasserversorgung handelt es sich allerdings um ein natürliches Monopol, das auf der Besonderheit des Gutes Wasser beruht. Zum einen macht der hohe Fixkostenanteil in der Wasserversorgung die Verlegung paralleler Netze durch konkurrierende Anbieter unrentabel. Damit stellen zumindest die Leitungsnetze ein natürliches Monopol dar, nicht dagegen die För-

1 Vgl. Meyer-Renschhausen, Martin, Die Auswirkungen der Privatisierung öffentlicher Dienstleistungen auf die Umwelt am Beispiel von Energiewirtschaft und Abwasserbeseitigung, in: Zeitschrift für öffentliche und gemeinwirtschaftliche Unternehmen, 1996, H. 1, S. 79-94 und S. 81. 
derung von Trinkwasser. Da jedoch Trinkwasser in unterschiedlichen Qualitäten bereitgestellt wird, handelt es sich um kein homogenes Gut, wie z. B. beim Strom, bei dessen Bereitstellung der Netzbetrieb durch einen Monopolisten von der Einspeisung durch konkurrierende Unternehmen getrennt werden kann. Die Durchleitung von Trinkwasser konkurrierender Anbieter durch das Trinkwasserversorgungsnetz ist wesentlich problematischer, da eine Durchmischung verschiedener Qualitäten hingenommen werden müsste. Daher sind Netzbetrieb und Trinkwasserproduktion nur schwer voneinander zu trennen. Ein Wettbewerb im Markt durch freien Leitungsbau oder durch gemeinsame Durchleitung verschiedener Anbieter durch fremde Trinkwassernetze ist somit kaum möglich, jedoch ein Wettbewerb um den Markt, bei dem die Gebietsmonopole erhalten bleiben und ihr Betrieb zeitlich befristet ausgeschrieben wird. Hinsichtlich der Modalitäten des Ausschreibungswettbewerbs und des Grades der Privatisierung unterscheiden sich die drei im Folgenden dargestellten Regulierungsmodelle deutlich.

\section{Regulierungsmodelle im internationalen Vergleich}

Im angelsächsischen Modell wird das Vorhandensein dauerhafter privater Monopole akzeptiert, wobei deren negative Auswirkungen durch externe Regulierung minimiert werden sollen. Die Aufsichtsbehörden der meisten Staaten dieses Typus von Regulierung begrenzen den Gewinn des Wasserunternehmens durch Regulierung der Kapitalrendite, die Preise werden dagegen nicht direkt reguliert. In England und Wales gibt die Aufsichtsbehörde allerdings Preisobergrenzen für Endverbraucher vor, die den Unternehmen erlauben sollen, angemessene Gewinne zu erwirtschaften. Der Staat befindet sich hier in einer konträren Position zu den Anbietern und muss Informationsasymmetrien hinnehmen. Darüber hinaus können Ineffizienzen in der Ressourcenallokation (Averch-Johnson-Effekt) ${ }^{2}$ auftreten. Die Vorteile dieses Modells liegen in der klaren Trennung zwischen den Aufsichtsbehörden und den von ihnen kontrollierten Gewässerbenutzern bzw. Unternehmen sowie in der Tatsache, dass die Gesetzgebung und die Regulierung auf der nationalen Ebene entwickelt und koordiniert werden. Da die Verwaltungsstruktur an den Flusseinzugsgebieten ausgerichtet ist, wird als Nebeneffekt auch eine integrierte Gewässerbewirtschaftung erleichtert. ${ }^{3}$

Im französischen Regulierungsmodell tritt an die Stelle einer dauernden, regulierenden Aufsicht in regelmäßigen Abständen ein Element des Wettbewerbs. Konzessionsverträge zwischen Kommunen und privaten Unternehmen regeln hier den Betrieb kommunaler Wasserdienstleistungen. Diese beinhalten i. d. R. ein ganzes Leistungspaket vom Betrieb der Wassernetze über die Finanzierung und die strategische Planung. Durch den Wettbewerb der privaten Anbieter um die Verträge können deren Gewinne begrenzt werden. Während der Laufzeit der Verträge findet allerdings wenig Wettbewerb statt. Im Gegensatz zum angelsächsischen Modell behalten die Kommunen hier einigen Einfluss auf die Entwicklung ihrer Infrastruktursysteme. Vor allem dieses

2 Überkapitalisierung im Gefolge der Regulierung der Profite, da eine Kapitalerhöhung die Basis erhöht, auf die die (regulierte) „rate of return on capital“ berechnet wird, vgl. Averch, H. Johnson, L.L., Behaviour of the Firm under Regulatory Constraint, in: American Economic Review, Vol. 52 (1962), S. 1052 -1069.

3 Vgl. Correia, Francisco Nunes und Andreas Kraemer, Institutionen der Wasserwirtschaft in Europa, Einleitung, Berlin, Heidelberg, 1997, S. 9. 
Regulierungsmodell führte zur Bildung großer, vertikal integrierter Wasser- und Bauunternehmen, die zugleich als Betreiber von Systemen der kommunalen Wasserversorgung und Abwasserbeseitigung und als Anbieter einschlägiger Güter und Leistungen auftreten.

Das deutsche Modell ist dagegen kein Regulierungsmodell im üblichen Sinne des Wortes Regulierung, da kein externes Verhältnis zwischen privatem Versorgungsunternehmen oder Betreibern und Behörde besteht. Anstelle einer Kontrolle natürlicher Monopole von außen wird hier der Einfluss auf den Betrieb von kommunalen Wassernetzen durch Eigentümerrechte ausgeübt und die Kommunen sind am Betrieb beteiligt. Informationsasymmetrien zwischen öffentlicher Verwaltung und Privatunternehmen treten daher kaum auf. Der Betrieb der Wassernetze wird streng von Herstellung und Angebot der mit den Dienstleistungen verbundenen Gütern getrennt: das Risiko von Ineffizienzen wird auf diese Weise verringert. Für den Betrieb der natürlichen Monopole werden kommunale Betriebe gegründet, in den anderen Segmenten der Wasserindustrie herrscht dagegen Wettbewerb. Die kommunalen Gesellschaften und Eigenbetriebe sind im deutschen Modell typisch für den Betrieb von Infrastuktursystemen der Wasserversorgung, ebenso die kommunale Zweckverbände, die eigens für diese Aufgabe gegründet wurden. Das deutsche Modell funktioniert im Wesentlichen ohne formale, externe Regulierung der Wasserpreise, Tarife oder Kapitalrenditen. Die Wassergebühren öffentlich-rechtlicher Betreiber unterliegen dem Kostendeckungsprinzip gemäß dem Kommunalabgabengesetz und die Wasserpreise der privatwirtschaftlichen Betreiber unterliegen der kartellrechtlichen Missbrauchsaufsicht.

Die Herausbildung der verschiedenen Regulierungsmodelle hängt eng mit der geschichtlichen Entwicklung des Wasserrechts in den verschiedenen Ländern zusammen. Grundsätzlich sind hinsichtlich der Entwicklung der rechtlichen Grundlagen der Trinkwasserversorgung drei Phasen zu unterscheiden. In der ersten, vorindustriellen Phase gingen die Rechtsordnungen in europäischen Ländern wie Frankreich, Großbritannien oder Deutschland von der scheinbar unbegrenzten Verfügbarkeit der Ressource Wasser aus. Konflikte aufgrund der Nutzung oder Verschmutzung von Gewässern waren im nachbarschaftlichen Bereich angesiedelt und wurden mit Mitteln des Bürgerlichen Rechts im Sinne des Ausgleichs privater Interessen beigelegt. In der zweiten, frühindustriellen Phase seit Beginn des 19. Jahrhunderts trat die Ver- und Zuteilung der knapper werdenden Ressource Wasser in den Vordergrund und die Wassergesetzgebung führte staatliche Genehmigungs- oder Erlaubnisvorbehalte ein. Damit erfolgte eine Verlagerung von der Individualbetrachtung zur öffentlichen Bewirtschaftung. Erst in der dritten Phase, welche in die zweite Hälfte des zwanzigsten Jahrhunderts fällt, kam es auf dem Höhepunkt von Industrialisierung und Urbanisierung sowie der damit hervorgerufenen Verschmutzung der Gewässer zu einer an den Zielen des Umweltschutzes ausgerichteten Wassergesetzgebung. Damit wurde die Bewirtschaftung der Gewässer auf die Erfordernisse des Gemeinwohls ausgerichtet. ${ }^{4}$

4 Vgl. hier und zu Folgendem: Breuer, Rüdiger, Wasserrecht in Mitgliedsstaaten der Europäischen Union, in: Correia, Francisco Nunes und R. Andreas Kraemer, Dimensionen Europäischer Wasserpolitik, Berlin, Heidelberg, 1997, S. 285 ff. 


\section{Herausbildung unterschiedlicher Privatisierungsformen}

\section{Die Vollprivatisierung}

In Großbritannien wurde der Gewässerschutz in der ersten Phase bis in das 19. Jahrhundert hinein vom „,common law“ geprägt. Im Mittelpunkt standen dabei das private Interesse der Eigentümer von Ufergrundstücken und die Lösung von Nachbarschaftskonflikten. Mit einsetzender Industrialisierung und Urbanisierung kam es 1876 zur Verabschiedung des Rivers Pollution Prevention Act, der Regelungen über den Abfluss von Haushalts- und Industrieabwässern enthielt und die förmliche Kontrolle und Genehmigung von Gewässerverunreinigungen durch Behörden vorschrieb; damit wurde die zweite Phase eingeleitet. 1951 erhielten mit dem Rivers Prevention of Pollution Act lokale Wasserbehörden die Aufgabe, mittels eines Genehmigungssystems die Reinheit der Gewässer zu erhalten oder wiederherzustellen. 1973 wurde die Wassergesetzgebung durch den Water Act 1973 neu geordnet, ergänzend trat der Control of Pollution Act 1974 hinzu. Damit wurden die über 1.600 örtlichen Wasserbehörden durch landesweit 10 Wasserbehörden ersetzt, die für sämtliche Aufgaben der Wasserwirtschaft einschließlich der Wasserversorgung und Abwasserbeseitigung zuständig waren (3. Phase). Mit dem Water Act 1989 wurde die Privatisierung der bis dahin staatlichen Wasserversorgung und Abwasserbeseitigung eingeleitet; er wurde 1991 durch den Water Industry Act (WIA) und den Water Resources Act (WAR) ersetzt. Der WIA regelt seitdem das Recht der privaten Wasserunternehmer und ihre Kontrolle durch staatliche Behörden. Der WAR spezifiziert die Aufgaben und Befugnisse der durch den Water Act 1989 gegründeten National Rivers Authority, eine Zentralbehörde, welche die zehn alten Wasserbehörden ersetzte.

Das Regulierungssystem folgt der ,,spezialisierten Regulierung“; es besteht aus jeweils einer gesonderten, unabhängigen Aufsichtsbehörde für Wasserentnahme und Abwassereinleitungen, für die Trinkwasserqualität und für Wasserpreise und Versorgungsbedingungen: ${ }^{5}$

- Die „Environment Agency“ überwacht die Wasserqualität von Flüssen und Badegewässern sowie die Umweltauswirkungen der Handlungen der Unternehmen.

- Das „Drinking Water Inspectorate“ befasst sich mit der Sicherstellung der Trinkwasserqualität.

- Das „Office of Water Services“ (OFWAT) setzt die Preise innerhalb eines vorgegebenen Minimums und Maximums, wobei es die Performance der einzelnen Versorger bewertet.

Die Preisregulierung beinhaltet, dass die Unternehmen ihre Durchschnittspreise pro Jahr um einen Faktor RPI + k erhöhen dürfen, wobei RPI den Einzelhandelspreisindex darstellt und k die zusätzlichen Kosten durch umwelt- und qualitätsbezogene Auflagen darstellt. Die Regulierungsbehörde setzt die Preise damit so, dass ein effizientes Unternehmen eine angemessene Verzinsung auf sein ursprüngliches Betriebsvermögen erwarten kann. ${ }^{6}$

Bei dieser Form der Privatisierung, wie sie in England und Wales vorzufinden ist, werden öffentlich betriebene Monopole als Ganzes auf einen privatwirtschaftlich orientierten Träger über-

5 Vgl. Kraemer, R. Andreas, Öffentliche und Private Wasserversorgung und Abwasserbeseitigung in Europa, in: Correia, Francisco Nunes und R. Andreas Kraemer, Dimensionen Europäischer Wasserpolitik, Berlin, Heidelberg, 1997, S. 292 f.

6 Vgl. Cowan, Simon, Competition in the Water Industry, in: Oxford Review of Economic Policy, Vol. 13, No.1, Spring 1997, S. $83-92$, S. 84. 
tragen. Deshalb spricht man hier auch von einer „Vollprivatisierung“. In Großbritannien erfolgte ein Verkauf der Betreiberfirmen inklusive aller Sachaktiva (wie z. B. Rohrleitungen, Kläranlagen und Wassergewinnungsanlagen) an private Investoren. In England und Wales wurden dabei zehn Wasserunternehmen geschaffen und deren Aktien öffentlich verkauft (in Schottland und Nordirland blieben Wasserversorgung und Abwasserentsorgung dagegen in öffentlicher Hand); daneben verblieben etwa 30 sondergesetzliche Wasserversorgungsgesellschaften, die früheren Verstaatlichungen entgangen waren und nun den gleichen Regulierungssystem wie die neuerdings privatisierten Unternehmen unterworfen wurden.

\section{Privatisierung durch Delegation}

In Frankreich traf der Code Civil von 1804 Regelungen über die private Nutzung der Gewässer, die der ersten wasserrechtlichen Entwicklungsphase zuzuordnen sind. Den Schritt zu einer administrativen, dem öffentlichen Interesse dienenden Gewässerbewirtschaftung stellt das Gesetz Nr. 64-1245 vom 16.12. 1964 betreffend den Wasserhaushalt, die Wasserverteilung und die Bekämpfung der Wasserverschmutzung dar, das die zweite Phase manifestierte. Zugleich wurde mit den Wasseragenturen ein neuer Verwaltungszweig zur Umsetzung der neuen Ziele geschaffen. Mit dem Inkrafttreten des Gesetzes Nr. 92-3 vom 3.1.1992, der loi sur l'eau, wurde das Wasser als Gemeingut der Nation definiert, dessen Schutz im allgemeinen Interesse liegt. Jede Handlung, die Wasser betrifft, ist seitdem entsprechend ihrer Auswirkung genehmigungs- oder erklärungsbedürftig. Für jedes Gewässereinzugsgebiet wurde ein Konzept von Wasserplanungs- und Bewirtschaftungsprogrammen eingeführt.

Durch das erste Wassergesetz im Jahre 1964 wurde Frankreich in sechs Einzugsgebiete aufgeteilt. In jedem dieser Flussgebiete wurden zwei Gremien gebildet:

- das Comité de Bassin (Komitee des Einzugsgebietes)

- die Agence de l'eau (Wasseragentur)

Das Comité de Bassin, ein lokales „Wasserparlament“ entscheidet über die Wasserpolitik und beschließt die Höhe der Abgaben. In diesem Gremium sind der Staat, die Regionen, die Départements und Gemeinden und die Wasser- bzw. Gewässerbenutzer vertreten. Der Präsident wird, wie die Mitglieder des Vorstandes der Agence, gewählt. Ziele und Prioritätensetzung für Maßnahmen sind in einem Wasserbewirtschaftungsleitplan (Schéma directeur d'aménagement et de gestion des eaux), der vom Comité de Bassin im Jahre 1996 verabschiedet wurde, festgelegt. Dieser Leitplan wurde durch das zweite Wassergesetz von 1992 vorgeschrieben.

Die Agence de l'eau ist eine staatliche Körperschaft öffentlichen Rechts, deren Präsident und Direktor vom Staat ernannt werden. Die Agence führt die wasserwirtschaftlichen Maßnahmen durch, erhebt Wasserabgaben für Wasserbenutzung und Wasserverschmutzung und erteilt Beihilfen für Investitionen und Reinigungsbetriebskosten. ${ }^{7}$

Die Höhe der Wassertarife wird durch das Comité de Bassin, in dem der Staat, die Regionen, die Départements und Gemeinden und die Wasser- bzw. Gewässerbenutzer vertreten sind, festgelegt.

7 Vgl. Langenfeld, F., Agence de l'Eau Rhin-Meuse, Metz, Das System der flussgebietsbezogenen Wasseragenturen in Frankreich, Vortrag auf dem Kongress „,Wasser Berlin 2000“, 23.-27.10.2000. 
Die wasserwirtschaftlichen Maßnahmen werden durch die Agence de l'eau, einer Körperschaft öffentlichen Rechts, angeordnet. Die Agence erhebt des Weiteren Wasserabgaben für Wasserbenutzung und Wasserverschmutzung und erteilt Beihilfen für Investitionen und Reinigungsbetriebskosten.

Durch die befristete Übertragung der Verantwortung für den Betrieb von Wassernetzen auf private Betreiber, wie sie in Frankreich praktiziert wird, kann der Betrieb bestehender Wasseranlagen zum einen für eine relativ kurze Zeit konzediert werden, wobei die Anlagen in der Regel in kommunalem Eigentum verbleiben. Zum anderen kann der Bau und der Betrieb von neu zu erstellenden Anlagen für längere Zeit an private Unternehmen übertragen werden, wobei am Ende des Zeitraums das Eigentum dann an die Kommune übergeht. Unter einem Konzessionsvertrag baut, finanziert und betreibt der Konzessionär bestimmte Anlagen für 20 bis 25 Jahre. Er enthält eine vertragliche Entlohnung, i. d. R. berechnet nach Kubikmeter Wasser oder Abwasser. ${ }^{8}$ Im Gegensatz zum angelsächsischen Privatisierungsmodell bleiben die Sachaktiva beim französischen Privatisierungsmodell im Eigentum der öffentlichen Hand, also des Staates bzw. der Kommunen oder Départements, lediglich die Betriebsverantwortung wird an Private delegiert. Die Betreiberlizenzen werden mittels eines Bietverfahrens vergeben.

\section{Privatisierung mit Regulierung durch die Aufsichtsorgane}

In Deutschland entwickelte sich die Wasserversorgung in der zweiten Phase als Bestandteil der kommunalen Daseinsvorsorge. In den im Gefolge der Industrialisierung während des 19. Jahrhunderts schnell wachsenden Städten lösten unzureichende hygienische Verhältnisse immer wieder Epidemien aus. Unter diesem Problemdruck begannen die Städte Einrichtungen der kommunalen Müllabfuhr, der zentralen Wasserversorgung und Abwasserbeseitigung aufzubauen. Zur Daseinsvorsorge für die Bürger wurden spezialisierte kommunale Einrichtungen geschaffen, die Umweltschutzaufgaben professionell erledigten und hygienische Mindeststandards kostengünstig sicherstellten. ${ }^{9}$

Diese zweite, frühindustrielle Entwicklungsphase des Wasserrechts hat sich vor allem in der Gesetzgebung der Länder niedergeschlagen. So gab es z. B. in Bayern bereits 1852 drei Gesetze über die Benutzung des Wassers, über die Bewässerungs- und Entwässerungsunternehmen sowie über den Uferschutz und den Schutz gegen Überschwemmungen. An der Schwelle von der zweiten zur dritten Entwicklungsphase steht das Wasserhaushaltsgesetz vom 27.7.1957 (am 1.3.1960 in Kraft getreten), das auf die Rahmengesetzgebung des Bundes gestützt ist und durch die Wassergesetze der Länder inhaltlich ausgefüllt wird.

In Deutschland ist die Zuständigkeit für die Wasserwirtschaft damit überwiegend bei den Ländern angesiedelt. Abgesehen von den Stadtstaaten Berlin und Hamburg haben alle Länder Vollzugsbehörden, denen technische Fachbehörden zugeordnet sind. Die Zuständigkeit für den Gewässerschutz und die Gewässerbewirtschaftung wird in den meisten Ländern auf mehreren Stufen verteilt. In den größeren Flächenstaaten sind dies

8 Vgl. Kraemer, R. A., a.a.O., S. S. 293 f.

9 Vgl. Hucke, J., Kommunale Umweltpolitik, in: Roth, R. und Wollmann, H.: Kommunalpolitik. Politisches Handeln in den Gemeinden, Bonn 1998, S. 657. 
- die oberste Wasserbehörde (i. d. R. das Umweltministerium) mit der Zuständigkeit für strategische Entscheidungen

- die obere, höhere oder mittlere Wasserbehörde, die in der Regel den Regierungspräsidien oder Bezirksregierungen zugeordnet ist und für die regionale Wasserwirtschaftsplanung zuständig ist und

- die untere Wasserbehörde (Städte, Stadt- und Landkreise sowie Wasserwirtschaftsämter) mit Überwachungs-, technischer Beratungs- und Vollzugsfunktion. ${ }^{10}$

Zum Zweck der Harmonisierung des Wasserrechts der Länder wurde des Weiteren die Länderarbeitsgemeinschaft Wasser (LAWA) eingerichtet, die von den obersten Wasserbehörden gebildet wird. Für die Koordination in der Bewirtschaftung von Flussgebieten haben die Länder Arbeitsgemeinschaften gebildet. ${ }^{11}$

Im Gegensatz zur Situation in der Bundesrepublik ähnelte die Struktur der Wasserversorgung in der früheren DDR, was die räumliche Ausdehnung und die Organisationsform betrifft, derjenigen in England. In den Jahren 1950 bis 1990 wurde die Wasserwirtschaft in der DDR von einer dezentralen, kommunalen Struktur mit rund 2.500 Vereinigungen, Zusammenschlüssen, Genossenschaften und $\mathrm{GmbHs}$ in eine zentralgeleitete regionale Monopolstruktur mit 15 volkseigenen Betrieben der Wasserversorgung und Abwasserbehandlung (WAB) übergeführt. Sieben Wasserwirtschaftsdirektionen übernahmen die Aufgaben der Gewässeraufsicht und -kontrolle, die 1972 mittlerweile auf 5 reduziert - gemeinsam mit den 15 WABs dem neu gebildeten Ministerium für Umweltschutz und Wasserwirtschaft unterstellt wurden. Damit waren faktische alle Organe der Wasserwirtschaft dem Umweltministerium untergeordnet; Kontrolle und Betrieb der Wasserwirtschaft lagen - ähnlich wie in England vor der Privatisierung - in einer Hand. ${ }^{12}$

Das deutsche Privatisierungsmodell sieht eine Regulierung der privatisierten Unternehmen durch deren Aufsichtsorgane vor. Durch Entsendung von Vertretern der öffentlichen Hand in die Aufsichtsorgane wird Einfluss auf die Geschäftspolitik der Wasserversorger ausgeübt; die Preisfestsetzung unterliegt wiederum der kartellrechtlichen Missbrauchsaufsicht. Es sind grundsätzlich zwei verschiedene Formen dieser Art von Privatisierung sowie eine Mischform zu unterscheiden: Die formale Privatisierung oder Organisationsprivatisierung: In diesem Fall verbleibt die Aufgabe der Wasserversorgung beim bisherigen Verwaltungsträger, lediglich wird die Trägerschaft in eine privatrechtliche Unternehmensform übergeführt, etwa bei der Umwandlung von einem Eigenbetrieb in eine Eigengesellschaft. Trotz formaler Privatisierung werden öffentlichrechtliche Strukturen aufrechterhalten, die jedoch in Bezug auf die Unabhängigkeit und Flexibilität der Betriebsführung privatrechtlichen Unternehmen nahe kommen soll.

Die materielle Privatisierung oder Aufgabenprivatisierung: Hier entledigt sich der Verwaltungsträger seiner Aufgabe vollständig zugunsten von Privaten. Diese Entäußerung des öffentlichen Aufgabenbestands kann widerrufbar oder endgültig sein. ${ }^{13}$ Eine Regulierung der privati-

10 Vgl. Kraemer, R. Andreas und Frank Jäger, Deutschland, in: Correia, F. N. und R. A. Kraemer, Institutionen der Wasserwirtschaft in Europa, Berlin, Heidelberg, 1997, S. 44.

11 Vgl. ebenda, S. $45 \mathrm{ff}$.

12 Vgl. Spelthahn, S., Privatisierung natürlicher Monopole - Theorie und internationale Praxis am Beispiel Wasser und Abwasser, Berlin 1993, S. $196 \mathrm{ff}$.

13 Vgl. Meyer-Renschhausen, M., a.a.O., S. $81 \mathrm{f}$. 
sierten Unternehmen erfolgt in beiden Fällen durch Einrichtung von Aufsichtsräten und Benennung von Aufsichtspersonen innerhalb der Unternehmen. ${ }^{14}$

Gemischtwirtschaftliche Form der Privatisierung oder Teilprivatisierung: Hierbei handelt es sich um eine Mischform zwischen formaler und materieller Privatisierung in Gestalt einer Beteiligung von Privatunternehmen an einer kommunalen Gesellschaft, die durch das sogenannte „Berliner Modell“ bekannt wurde. Bei der Teilprivatisierung der Berliner Wasserbetriebe (BWB) wurde ein Holding-Modell gewählt, bei dem das Land Berlin 50,1\% der Anteile an der strategischen Steuerungsholding Berlinwasser Holding Aktiengesellschaft hält. Die restlichen 49,9\% der Aktien der Berlinwasser Holding Aktiengesellschaft hält eine Beteiligungsaktiengesellschaft, die von einem Investorenkonsortium, bestehend aus RWE, Vivendi und Allianz gegründet wurde, das zugleich eine stille Beteiligung an der Holding einging. Der Gesellschaftszweck der Holding ist die Steuerung und Fortentwicklung des Wettbewerbsgeschäftes und die Steuerung der Berliner Wasserbetriebe. Die Rechtsform der BWB als Anstalt öffentlichen Rechts blieb dabei unverändert, ihr wurden aber die Wettbewerbsgesellschaften ausgegliedert, die nun in die Berlinwasser Holding Aktiengesellschaft eingebracht wurden. Durch diese Teilprivatisierungskonstruktion wurde der Einfluss des Landes Berlin auf die BWB und die Holding Aktiengesellschaft gewährleistet, gleichzeitig blieb wegen der stillen Beteiligungen der Hoheitsbetrieb Entwässerung steuerfrei. $^{15}$

\section{Wettbewerbsstrukturen in der Wasserwirtschaft}

Will man die Auswirkungen der verschiedenen Regulierungs- und Privatisierungsformen auf die Wettbewerbsstruktur richtig einordnen, so muss man zwischen der kommunalen Wasserversorgung einerseits und der Wasserindustrie andererseits unterschieden. Erstere fällt wie die kommunale Abwasserbeseitigung in die Kategorie der öffentlichen Dienstleistungen (auch wenn sie von Privaten erbracht werden). Der Begriff der Wasserindustrie bezieht sich dagegen auf die mit der Wasserversorgung und Abwasserbeseitigung verbundenen Waren und Dienstleistungen; er schließt z. B. die Herstellung von Rohren, Pumpen, Filtern, mess- und regeltechnischem Gerät ein. Einige der großen Wasserunternehmen, vor allem in Frankreich, betreiben kommunale Wassersysteme und sind zugleich Hersteller von Waren und Anlagen für die Wasserversorgung und Abwasserbeseitigung.

Die drei im vorangegangenen Abschnitt beschriebenen Privatisierungsmodelle führen zu drei klar unterscheidbaren Formen des Wettbewerbs:

- „Ersatzwettbewerb“ zwischen privaten Versorgungs-Unternehmen, der von Regulierungsbehörden simuliert wird (,Yardstick Competition“").

- Wettbewerb zwischen privaten Betreibern um das Recht zum befristeten Betrieb natürlicher Monopole (,Franchise bidding“).

- Wettbewerb in den Waren- und Dienstleistungs-Märkten im Wasserbereich.

14 Vgl. Kraemer, R. A., a.a.O., S. 297.

15 Vgl. Mecke, Thomas, Das Berliner Modell - Weltweit gültig? Vortrag auf dem Kongress „Wasser Berlin 2000“, 23.-27.10.2000. 
Im angelsächsischen Modell gibt es keinen direkten Wettbewerb unter den privaten Versorgungsunternehmen, weder um die Verbraucher, noch um deren Versorgungsgebiete. Der „Ersatzwettbewerb" erfolgt in Form eines Benchmarkings der Versorgungsunternehmen, das die Regulierungsbehörde vornimmt. Die Regulierungsbehörde benötigt dabei auch Informationen über die Märkte für Waren und Leistungen im Wasserbereich, denn private Versorgungsunternehmen und Betreibergesellschaften, die zugleich Anbieter dieser Güter sind, können regionale oder sektorale Monopole oder Kartelle aufbauen um Gewinne in nicht regulierte Bereiche zu verschieben. Das gilt auch für das französische Modell, wo ein Wettbewerb um den befristeten Betrieb örtlicher Wasserversorgungssysteme in Gestalt eines Bietverfahrens stattfindet. Im deutschen Modell gibt es dagegen keinen direkten Wettbewerb zwischen den kommunalen Trägern, diese halten ihre natürlichen Monopole auf der regionalen Ebene aufrecht. Leistungsvergleiche zwischen den verschiedenen Anbietern werden von den kommunalen Betrieben selbst vorgenommen. Dagegen ist der Wettbewerb bei den Gütern im Wasserbereich intensiv, diese Märkte sind durch eine Vielzahl kleiner und mittlerer Betriebe gekennzeichnet. Versorgungsunternehmen und Wasserindustrie sind damit im Wesentlichen zwei klar voneinander abgrenzbare Gruppen. ${ }^{16}$

Im französischen Modell findet der Wettbewerb im Rahmen der Ausschreibungen ,ex-ante“ statt. Dabei erfolgt eine befristete Übertragung des Betriebs der Wassernetze auf private Betreiber durch eine Ausschreibung der Leistungserstellung durch die Kommunen. Die Anlagen zur Wasserversorgung bleiben im kommunalen Eigentum, lediglich

der Betrieb der Versorgungsnetze wird delegiert. Soweit es sich bei den Verträgen zwischen Kommunen und Wasserversorgungsunternehmen um Pachtverträge handelt, bei denen die erforderlichen Investitionen - anders als beim Konzessionsvertrag - im Wesentlichen von den Kommunen übernommen werden, entfallen die Kapitalbewertungsprobleme von langlebigen Anlagen, welche für die Unternehmen den freien Marktein - und austritt behindern würden. Dies gestattet die Wiederholung des Auktionsverfahrens in relativ kurzen Zeitabständen von 10 bis 15 Jahren beim Pachtvertrag. Beim Konzessionsvertrag betragen die Laufzeiten aufgrund der versunkenen Kosten in Neuinvestitionen, die der private Betreiber übernimmt, 20 bis 30 Jahre. Eine dritte Möglichkeit stellt der Betriebsführungsvertrag dar, bei dem nur Teilleistungen auf Private übertragen werden mit einer Vertragsdauer von 6 bis 10 Jahren. In den jeweiligen Verträgen wird festgelegt, welche Kosten der Private maximal in Rechnung stellen darf und die vertragliche Entlohnung geregelt. ${ }^{17}$

Für Frankreich ist die hohe Bedeutung der privatwirtschaftlichen Wasserversorgung auf die geschichtliche Entwicklung zurückzuführen. Das Gut Wasser wurde schon sehr früh als normales „kommerzielles“ Gut angesehen und nicht wie in Deutschland als Gegenstand der öffentlichen Daseinsvorsorge. Auch sind die im Vergleich zu Deutschland wesentlich kleineren Gemeinden aufgrund mangelnden Fachpersonals und fehlendem Fachwissen gar nicht in der Lage, die Trinkwasserversorgung selbst durchzuführen. ${ }^{18}$ Bereits im 19. Jahrhundert wurden private Wasserversorgungsunternehmen gegründet: 1853 die Société Générale des Eaux und 1880 die Lyonnaise des Eaux. 1933 wurde die drittgrößte Gruppe, die Société d'Amenagement Urbain et Rural

16 Vgl. Kraemer, R. A., a.a.O., S. 298 f.

17 Vgl. Spelthahn, S., a.a.O., S. $154 \mathrm{f}$.

18 Vgl. Spelthahn, S., a.a.O., S. 156 f. 
(SAUR) gegründet. ${ }^{19}$ Diese Unternehmensgruppen bzw. ihre Rechtsnachfolger haben heute im internationalen Geschäft die größten Marktanteile, was darauf beruht, dass unter ihrem Dach alle für komplexe wasserwirtschaftliche Projekte erforderlichen Komponenten (Anlagenfertigung, Engineering, Hoch- und Tiefbau, Entwicklungsabteilungen) vorhanden sind.$^{20}$ In Großbritannien sind die bis dahin öffentlichen Einrichtungen der Wasserver- und Abwasserbeseitigung 1989 in einer großangelegten nationalen Aktion privatisiert worden. So entstanden die Water Service Companies unter dem Dach großer regionaler Holding-Gesellschaften; darunter waren mehrere Anbieter, wie Thames Water, Severn Trent Water, Anglian Water und United Utilities, international tätig. ${ }^{21}$

In Deutschland dagegen ist die wasserwirtschaftliche Kompetenz eindeutig auf der kommunalen Ebene angesiedelt, dies geht allerdings zu Lasten der internationalen Wettbewerbsfähigkeit. Ein Vorteil der Strukturen in der deutschen Wasserversorgung beruht darauf, dass die starke kommunale Verankerung der deutschen Wasserversorgungsunternehmen ein hohes Maß an politischer Beteiligung sicherstellt. Damit wird die Vorsorgeorientierung der Gewässerbewirtschaftung auf eine solide Grundlage gesetzt. Dieses System hat bisher starke Akzeptanz in der Bevölkerung gefunden. Das hohe Niveau und die Effizienz in der technischen Betriebsführung werden durch die enge Kooperation zwischen Wasserversorgungsunternehmen, Industrie, staatlichen Stellen sowie durch die Aktivität der regelsetzenden technisch-wissenschaftlichen Vereine gewährleistet. Aufgrund der starken funktionalen und organisatorischen Fragmentierung ist der Einfluss der deutschen Wasserwirtschaft auf die Entscheidungsprozesse in der Europäischen Union jedoch nur gering. ${ }^{22}$ Durch die stark arbeitsteilige Organisation (Wasserver- und Abwasserentsorgungsunternehmen, Bauunternehmen, Anlagenbauer, Komponentenlieferanten, Consulting-Firmen, Ingenieurbüros, Wasserlaboratorien und Forschungsinstitute) fehlt der deutschen Wasserwirtschaft das einheitliche Auftreten auf den internationalen Märkten, weswegen das ständig wachsende Marktsegment der Paketlösungen nicht besetzt werden kann. ${ }^{23}$

Vor allem das französische Modell führte zur Bildung großer, vertikal integrierter Wasser- und Bauunternehmen, die zugleich als Betreiber von Systemen der kommunalen Wasserversorgung und Abwasserbeseitigung und als Anbieter einschlägiger Komponenten, Bau- und Dienstleistungen auftreten. Die drei führenden französischen Unternehmen Suez, Veolia und Saur sind - neben ihren weltweiten Aktivitäten - in einer Reihe von europäischen Ländern tätig. Daneben sind zwei spanische Wasserversorger, bis 2008 auch die britische United Utilities und die österreichische Energie AG europaweit vertreten. Für Deutschland ist das Wasserversorgungsunternehmen Gelsenwasser zu nennen, das außer im Heimatland allerdings nur noch in Polen und Ungarn und mit einer kleinen Beteiligung in Frankreich (Nantaise des Eaux) präsent ist (vgl. Tabelle 1). Als weitere deutsche Unternehmen sind zwar auch RWE und Berlinwasser in Osteuropa vertreten, jedoch jeweils in enger Bindung an Veolia oder Suez. RWE hat bereits 2004 mit dem Verkauf von Thames Water und seiner Mehrheitsbeteiligung an American Water Works den Rückzug vom internatio-

19 Vgl. Spelthahn, S., a.a.O., S. 128.

20 Vgl. BMBF (Hrsg.) (2000) Aktionskonzept nachhaltige und wettbewerbsfähige deutsche Wasserwirtschaft, Karlsruhe, März 2000, S. 76.

21 Vgl. BMBF, a.a.O., S. 77.

22 Vgl. BMBF, a.a.O., S. 19.

23 Vgl. BMBF, a.a.O., S. $69 \mathrm{ff}$. 
nalen Wassergeschäft eingeleitet. Berlinwasser ist darüber hinaus noch mit seiner internationalen Abteilung Berlinwasser International (BWI) mit Beratungs- Engineering- und kurzfristigen Managementprojekten in Osteuropa, Asien und Südamerika vertreten. Insgesamt hat sich die Konzentration auf dem europäischen Wassermarkt bis zum Jahr 2010 weiterhin fortgesetzt, und zwar unter dem Vorzeichen einer überwältigenden Dominanz von Suez und Veolia. Allerdings gab es in den vorangegangenen drei Jahren keine größeren Privatisierungen von Wasserdienstleistungen mehr. ${ }^{24}$

\begin{tabular}{|c|c|c|}
\hline Unternehmen & Hauptsitz & Niederlassungen in Europa \\
\hline Suez & Frankreich & $\begin{array}{c}\text { Frankreich, Deutschland, Großbritannien, } \\
\text { Italien, Spanien, Tschechische Republik, } \\
\text { Slowakei, Ungarn, Rumänien }\end{array}$ \\
\hline Veolia & Frankreich & $\begin{array}{c}\text { Frankreich, Deutschland, Großbritannien, } \\
\text { Italien, Tschechische Republik, Slowakei, } \\
\text { Estland, Rumänien, Bulgarien }\end{array}$ \\
\hline Saur/Séché & Frankreich & Frankreich, Polen \\
\hline FCC/Aqualia & Spanien & $\begin{array}{c}\text { Spanien, Italien, Portugal, Tschechische } \\
\text { Republik }\end{array}$ \\
\hline Sacyr Vallehermosa/Valoriza & Spanien & Spanien, Portugal \\
\hline United Utilities & Großbritannien & $\begin{array}{c}\text { Großbritannien und bis 2008: Estland, Bul- } \\
\text { garien, Polen }\end{array}$ \\
\hline Energie AG & Österreich & $\begin{array}{c}\text { Österreich, Tschechische Republik, Slowe- } \\
\text { nien }\end{array}$ \\
\hline Gelsenwasser & Deutschland & $\begin{array}{c}\text { Deutschland, Frankreich, Tschechische } \\
\text { Republik, Polen }\end{array}$ \\
\hline RWE & Deutschland & Deutschland, Polen, Ungarn, Kroatien \\
\hline Berlinwasser & Deutschland & Deutschland, Ungarn, Polen \\
\hline
\end{tabular}

Tabelle 1: Europäische Wasserversorger mit Geschäftstätigkeit in mehr als einem Land 2010

Quelle: Hall/Lobina (2010), S. 4, 20

Dagegen fanden bedeutende Fälle von Re-Kommunalisierungen statt. So entschied sich die französische Hauptstadt Paris nach Auslaufen des 25-jährigen Konzessionsvertrags mit Suez and Veolia für die Re-Kommunalisierung der Wasserversorgung und erteilte dem öffentlichen Betreiber Eau de Paris den Zuschlag. ${ }^{25}$ Insgesamt ist europaweit ein zunehmender Widerstand gegen die Privatisierung der Wasserversorgung festzustellen. In Italien verabschiedete das Parlament 2007 ein einjähriges Moratorium gegen die Privatisierung der Wasserversorgung und brachte einen Gesetzesentwurf zur Verhinderung weiterer Privatisierungen ein. In Nordirland gab es eine erfolgreiche Kampagne gegen die Privatisierung der Wasserversorgung. ${ }^{26}$

24 Vgl. Hall, David and Emanuele Lobina, Water Companies in Europe 2010, London, September 2010.

25 Vgl. ebenda.

26 Vgl. David Hall and Emanuele Lobina, Water in Europe, January 2008, sowie Library of the European Parlament, Library Briefing 10/2/2011 - "Water privatisation in the EU". 


\section{Fazit}

Abschließend bleibt festzuhalten, dass jedes der drei grundlegenden Regulierungsmodelle seine jeweiligen Vor- und Nachteile aufweist. So beruht der Vorteil des angelsächsischen Regulierungsmodells auf der klaren Trennungslinie zwischen den Aufsichtsbehörden und den von ihnen kontrollierten Gewässerbenutzern bzw. Unternehmen, der Koordinierung der Gesetzgebung und Regulierung auf der nationalen Ebene sowie der Orientierung der Verwaltungsstruktur an den Flusseinzugsgebieten, die eine integrierte Gewässerbewirtschaftung erleichtert. Als Nachteile sind anzuführen, dass der Staat Informationsasymmetrien hinnehmen muss und sich einem hohen Informationsaufwand bezüglich der Märkte für Waren und Dienstleistungen im Wasserbereich gegenüber sieht. Daher besteht grundsätzlich die Gefahr der Gewinnverschiebung in nicht regulierte Geschäftsbereiche.

Vor allem das französische Regulierungsmodell führte zur Bildung großer, vertikal integrierter Wasserunternehmen, die zugleich als Betreiber von Systemen der kommunalen Wasserversorgung und Abwasserbeseitigung und als Anbieter einschlägiger Güter, Bau- und Dienstleistungen auftreten, woraus erhebliche internationale Wettbewerbsvorteile resultieren. Zwar begrenzt der Wettbewerb der privaten Anbieter um die Betreiber-Lizenzen ihre Gewinne, aber während der Laufzeit der Verträge, die bis zu 30 Jahre betragen kann, besteht kaum mehr Wettbewerb. Darüber hinaus können private Versorgungsunternehmen und Betreibergesellschaften, die zugleich Anbieter der korrespondierenden Güter sind, Gewinne in nicht regulierte Geschäftsbereiche verschieben.

In Deutschland ist dagegen der Betrieb der Wassernetze streng von Herstellung und Angebot der mit den Dienstleistungen verbundenen Güter getrennt. Die Versorgungsunternehmen einerseits und die Wasserindustrie andererseits sind klar voneinander abgrenzbar, was unter wettbewerbspolitischen Aspekten zu begrüßen ist. Die regionalen Versorgungsmonopole bleiben zwar auch im Falle der Privatisierung erhalten, in der Wasserindustrie herrscht dagegen intensiver Wettbewerb durch eine Vielzahl mittelständischer Betriebe, womit das Risiko von Ineffizienzen verringert wird. Die starke kommunale Verankerung der Wasserversorgung, aber auch Fragmentierung der Wasserwirtschaft erschweren aber ein einheitliches Auftreten auf internationalen Märkten. Große vertikal integrierte Unternehmen haben international einen klaren Wettbewerbsvorsprung bei Komplettlösungen, und die Trennung von Infrastruktur und Betrieb scheint die internationale Performance noch zu verbessern. Dagegen hat die stark fragmentierte deutsche Wasserindustrie bei Komplettlösungen erhebliche Wettbewerbsnachteile. Ein Aufholwettbewerb erscheint daher kaum Erfolg versprechend zu sein, zumal anzunehmen ist, dass ihm die bewährten Strukturen der Wasserversorgung in Deutschland geopfert werden müssten. 
Die deutsche Wasserwirtschaft im europäischen Vergleich

Abstract

Johann Wackerbauer; The German Water Management in an European Comparison

Water Management; Natural Monopoly; Privatisation; Liberalisation; Industry Structure, Ownership

In an international comparison, there are three basic models for the regulation of natural monopolies in the public water supply: the Anglo-Saxon, the French and the German models. The delimitation between supervisory bodies and operations in the water supply is strongest in the first model and weakest in the last. This has led to three basic types of privatisation: "full privatisation", "privatisation through delegation" and "privatisation with regulation by the supervisory bodies". These privatisation types go along with three clearly distinguishable forms of competition: substitute competition simulated by the regulation authorities between private supply-enterprises ("yardstick competition"), competition between private operators for the right to the temporary provision of water supply ("franchise bidding"), and competition in the product and service markets in the provision of water.

\section{Literaturverzeichnis}

Averch, H. Johnson, L.L., Behaviour of the Firm under Regulatory Constraint, in: American Economic Review, Vol. 52 (1962), S. $1052-1069$.

BMBF (Hrsg.), Aktionskonzept nachhaltige und wettbewerbsfähige deutsche Wasserwirtschaft, Karlsruhe, März 2000.

Breuer, Rüdiger, Wasserrecht in Mitgliedsstaaten der Europäischen Union, in: Correia, Francisco Nunes und R. Andreas Kraemer, Dimensionen Europäischer Wasserpolitik, Berlin, Heidelberg, 1997.

Correia, Francisco Nunes und Andreas Kraemer, Institutionen der Wasserwirtschaft in Europa, Einleitung, Berlin, Heidelberg, 1997.

Cowan, Simon, Competition in the Water Industry, in: Oxford Review of Economic Policy, Vol. 13, No.1, Spring 1997, S. 83-92, S. 84.

Hall, David and Emanuele Lobina, Water Companies in Europe 2010, A report commissioned by the European Federation of Public Service Unions (EPSU), London, September 2010.

Hall, David and Emanuele Lobina, Water in Europe, A report by the Public Services International Research Unit (PSIRU) in the Business School at the University of Greenwich, London, January 2008.

Hucke, J., Kommunale Umweltpolitik, in: Roth, R. und Wollmann, H.: Kommunalpolitik. Politisches Handeln in den Gemeinden, Bonn 1998.

Kraemer, R. Andreas, Öffentliche und Private Wasserversorgung und Abwasserbeseitigung in Europa, in: Francisco Nunes Correia, R. Andreas Kraemer, Dimensionen Europäischer Wasserpolitik, Berlin, Heidelberg, 1997.

Langenfeld, F., Agence de l'Eau Rhin-Meuse, Metz, Das System der flussgebietsbezogenen Wasseragenturen in Frankreich, Vortrag auf dem Kongress „Wasser Berlin 2000“, 23.-27.10.2000.

Library of the European Parlament, Library Briefing 10/2/2011 "Water privatisation in the EU”.

Mecke, Thomas,Das Berliner Modell - Weltweit gültig? Vortrag auf dem Kongress „Wasser Berlin 2000“, 23.-27.10.2000.

Meyer-Renschhausen, Martin, Die Auswirkungen der Privatisierung öffentlicher Dienstleistungen auf die Umwelt am Beispiel von Energiewirtschaft und Abwasserbeseitigung, in: Zeitschrift für öffentliche und gemeinwirtschaftliche Unternehmen, 1996, H. 1, S. 79-94.

Spelthahn, Sabine, Privatisierung natürlicher Monopole - Theorie und internationale Praxis am Beispiel Wasser und Abwasser, Berlin 1993. 